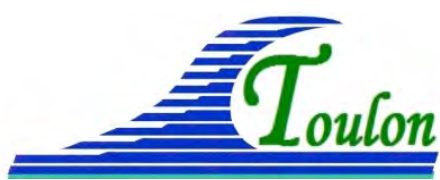

XIV èmes Journées Nationales Génie Côtier - Génie Civil

Toulon, 29 juin au $1^{\text {er }}$ juillet 2016

DOI:10.5150/jngcgc.2016.067 @ Editions Paralia CFL

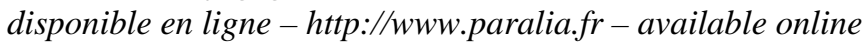

\title{
Analyses coûts-bénéfices (ACB) littorales
}

\section{Céline PERHERIN ${ }^{1}$, Natacha CHRISTIN ${ }^{2}$, Yann DENIAUD ${ }^{1}$, Delphine ROUCHON ${ }^{2}$}

1. Centre d'Études et d'expertise sur les Risques, l'Environnement, la Mobilité et l'Aménagement (Cerema/DTecEMF/DI/IE/IAR), Technopôle Brest Iroise - 155, rue Pierre Bouguer - BP 5 - 29280 Plouzané, France. Celine.Perherin@cerema.fr ; Yann.Deniaud@cerema.fr

2. Commissariat Général au Développement Durable (CGDD/SEEI/ERNR2), Tour Sequoia 92055 La Défense Cedex, France. \{Natacha.Christin ; Delphine.Rouchon\}@developpement-durable.gouv.fr

\section{Résumé :}

Les analyses coût/bénéfice (ACB), qui ont pour objectif d'estimer la rentabilité économique d'un projet, sont des outils d'aide à la décision pouvant être mobilisés pour la définition des stratégies de prévention des risques dans le cadre notamment de la labellisation d'un Programme d'Actions de Prévention des Inondations (PAPI), des demandes de financements au titre du Plan Submersions Rapides (PSR) ou de la mise en place de stratégies locales de gestion intégrée du trait de côte. Les ACB ont été développées initialement pour les projets de prévention des inondations par débordements de cours d'eau. Les ACB littorales sont confrontées à des difficultés méthodologiques. Le guide "Analyse Multi-Critères" élaboré par le CGDD aborde les spécificités littorales et propose une méthodologie pour les ACB littorales.

Les méthodes de détermination des aléas recul du trait de côte et submersion marine sont précisées. Pour que l'ACB puisse jouer son rôle, l'impact des ouvrages sur les aléas doit être finement défini. Pour la submersion marine, deux niveaux de mise en œuvre d'une ACB sont proposés suivant l'avancée du projet. Le choix de l'intensité des événements théoriques à étudier pour construire la courbe dommages-fréquences est abordé en lien avec le comportement des ouvrages de protection (niveaux de protection et de sûreté) et l'évolution des dommages aux enjeux. L'élévation du niveau marin lié au changement climatique nécessite l'élaboration de plusieurs courbes dommagesfréquences. Des recommandations méthodologiques sont également proposées (CEREMA, 2015) pour le recul du trait de côte, aléa non abordé dans les documents antérieurs. Au sujet de l'évaluation des dommages potentiels, le caractère salin de l'eau accentuant le montant des sinistres par rapport à des dommages en eau douce et la valorisation monétaire des biens et activités pouvant disparaître du fait du recul du trait de côte font l'objet de recommandations spécifiques.

Mots-clés : Analyse coûts-bénéfices, Aléas littoraux, Risques littoraux, Aménagements, Rentabilité économique. 


\section{Introduction}

Les analyses coûts-bénéfices (ACB) d'un projet d'aménagement et de ses variantes permettent d'apprécier leur pertinence économique. Elles sont réalisées sur un horizon temporel cohérent avec le projet d'aménagement, dans la limite de 50 ans. Ces outils d'aide à la décision permettent d'étudier notamment l'opportunité de stratégies de prévention des risques. Les ACB ont été rendues obligatoires dans certaines conditions dans le cadre de deux démarches: les Programmes d'Actions de Prévention des Inondations (PAPI) (MEDDTL, 2011) et le Plan Submersions Rapides (PSR) (MEDDTL, MIOCT, MINEFI, MBCPFPRE, 2011). La labellisation d'un PAPI, qui permet au porteur du projet de bénéficier de fonds publics, exige l'élaboration d'une analyse coûts-bénéfices des mesures de protection envisagées lorsque la somme des montants de ces mesures est supérieure à 2 millions d'euros ou représente $25 \%$ du montant total du PAPI. Dans le cadre de la labellisation PSR, l'ACB est obligatoire pour les ouvrages dont il est envisagé d'augmenter le niveau de protection. Les projets littoraux se placent également dans un contexte lié à la mise en place de la stratégie nationale de gestion intégrée du trait de côte (MEDDTL, 2012).

\section{Méthode d'élaboration des ACB}

\subsection{Cadrage général}

La méthode ACB, telle qu'exigée par le Ministère de l'Environnement, est définie dans le cahier des charges PAPI (MEDDTL, 2011). Un guide méthodologique, conforme au cahier des charges PAPI, les Annexes techniques (MEDDTL, 2010), fait une synthèse des outils jugés les plus adaptés pour la réalisation d’une ACB. Un guide général s'adressant spécifiquement aux porteurs de projet permet de faciliter l'appropriation des démarches d'ACB, leur compréhension et l'exploitation de cet outil qui favorise la structuration de stratégies pertinentes de gestion du risque d'inondation (CEPRI, 2011). Ces trois documents développent des outils adaptés pour l'étude des stratégies de gestion de l'aléa inondation par débordement de cours d'eau uniquement.

Face à un territoire exposé à des inondations, l'une de ces stratégies consiste à protéger le territoire, grâce à la mise en œuvre de mesures structurelles où les digues ont une place centrale. Des mesures de relocalisation peuvent également être envisagées. Ces mesures sont généralement à l'origine de bénéfices pour le territoire sous la forme de dommages évités aux logements, aux activités, aux équipements publics et aux réseaux. Inversement, ces mesures représentent aussi des coûts correspondant aux études préalables, aux travaux, à leur maintenance et à leur exploitation.

L’objectif de l'analyse coûts-bénéfices est de comparer, dans un cadre précis, ces bénéfices et ces coûts afin de tester la pertinence économique du projet, la mesure étant jugée pertinente si la somme des bénéfices excède les coûts. L’ACB nécessite donc d'évaluer les dommages potentiellement évités grâce au projet, en estimant les 


\section{XIV èmes Journées Nationales Génie Côtier - Génie Civil \\ Toulon, 29 juin au $1^{\text {er }}$ juillet 2016}

dommages pour la situation de référence (état initial) et les dommages pour les situations aménagées. À défaut de considérer la totalité des dommages potentiels, ce qui reste difficile d'un point de vue méthodologique, ce sont les dommages directs tangibles que la courbe de dommages de référence comptabilise principalement. Une étude de sensibilité obligatoire permet d'estimer l'impact des incertitudes.

Le calcul des bénéfices doit se faire pour l'ensemble des événements d'inondation pouvant survenir sur le territoire, quelle que soit leur fréquence et leur intensité. Les ACB de mesures de protection contre les inondations reposent ainsi sur l'élaboration de courbes dommages-fréquences (voir figure 1). Pour cela, un Dommage Moyen Annualisé (DMA) est calculé : ce DMA prend en compte les dommages engendrés par tous les événements (rares à fréquents) en pondérant ces dommages par leur probabilité d'occurrence annuelle. Sur le même principe, un Dommage Évité Moyen Annuel (DEMA) est calculé par soustraction du DMA en situation "avec projet" au DMA en situation de référence (voir figure 1).

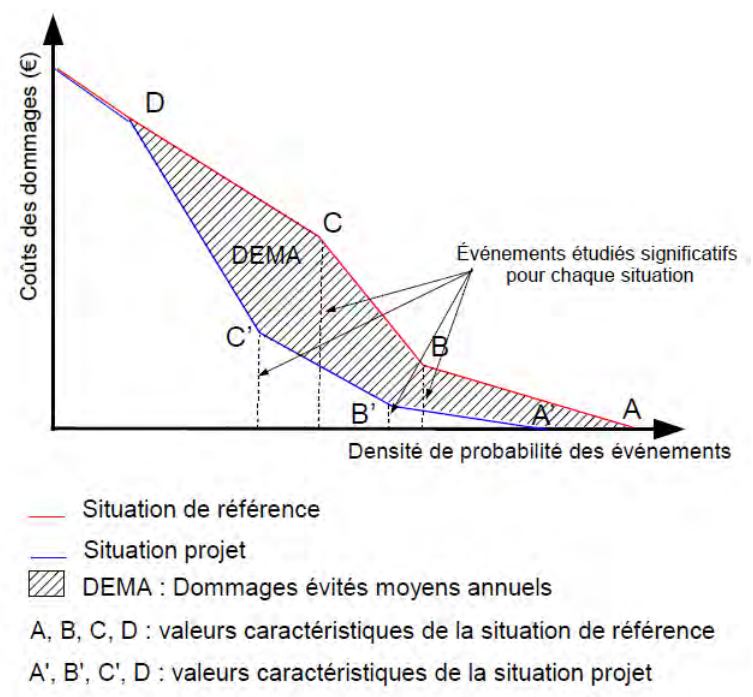

Figure 1. Définition des dommages évités moyens annuels (DEMA) (CEREMA, 2015).

La comparaison des bénéfices (DEMA) et des coûts du projet $\left(\mathrm{C}_{\mathrm{i}}\right)$ doit aussi se faire sur un horizon temporel fixé (n), de 50 ans maximum : une actualisation de ces valeurs, qui s’échelonnent dans le temps, est donc nécessaire. Le résultat de l’ACB se présente sous la forme d'une Valeur Actualisée Nette (VAN) (1) :

$V A N=-C_{0}+\sum_{i=0}^{n}\left(\frac{1}{(1+r)^{i}}\right)\left(D E M A-C_{i}\right)$

où : $C_{0}$ est le coût initial de la mesure (ici au temps $\mathrm{i}=0$ ), $D E M A$ les dommages évités moyens annuels, $C_{i}$ les coûts de fonctionnement et de maintenance du projet (à l'année i), $n$ l'horizon temporel de la mesure, $r$ le taux d'actualisation (2.5\% jusqu'en 2070, 1.5\% ensuite, recommandation du CGSP de 2013). 


\section{Thème 6 - Gestion durable des zones littorales et estuariennes}

Pour être pertinent économiquement, le projet doit présenter une VAN positive. Cependant, les données d'entrée et les hypothèses prises lors de ces analyses peuvent très fortement impacter les résultats. Il est ainsi recommandé de réaliser des analyses de sensibilité pour tester la robustesse de l'étude (CEPRI, 2011) par rapport aux incertitudes pesant sur la construction des fonctions de dommages et celles des modèles hydrauliques.

\subsection{Difficultés d'application au littoral}

Les ACB littorales sont confrontées à des difficultés méthodologiques spécifiques, concernant la détermination de l'aléa et l'évaluation des dommages potentiels. Les projets relatifs à la submersion marine ont de fortes particularités. L'espace littoral est mobile d'un point de vue hydro-sédimentaire, de sorte que les aléas de submersion marine et de recul du trait de côte, qui peuvent fortement interagir, doivent être étudiés simultanément. L'impact du changement climatique sur les aléas littoraux est important, du fait notamment de l'élévation du niveau marin moyen. Les modes d'entrée des eaux sont liés à des sollicitations hydrauliques spécifiques au milieu littoral, notamment à l'impact des vagues. La submersion marine s'avère plus dommageable que les crues de plaine aux montées des eaux lentes.

L’aléa littoral peut aussi impliquer la survenue de dommages supplémentaires de ceux rencontrés pour des inondations fluviales, notamment du fait du caractère salin de l'eau, accentuant le montant des sinistres par rapport à des dommages en eau douce. La valorisation monétaire des biens et activités pouvant disparaître du fait du recul du trait de côte appelle également des recommandations spécifiques.

La prise en compte de ces spécificités nécessite de proposer des méthodologies adaptées. Les recommandations pour les ACB littorales (MEDDE, 2014 ; CEREMA, 2015) ont été élaborés dans le cadre d'un groupe de travail faisant suite à une analyse et une comparaison d'études ACB littorales menées dans le cadre de projets PAPI ou PSR (PERHERIN et al., 2013).

\section{Principes d'analyses des aléas littoraux dans le cadre des ACB}

\subsection{Une démarche identique aux autres études d'aléas littoraux}

Les ACB reposent sur une analyse des dommages évités par les mesures de protection. Afin d'identifier ces dommages, les caractéristiques des aléas (inondation ou recul du trait de côte) touchant chaque catégorie d'enjeux doivent être parfaitement connues, et cela pour chacune des situations étudiés (situation de référence ou situations de projet). Une étude d'aléa complète doit ainsi être menée afin d’obtenir la précision nécessaire à l'étude de l'impact d'un ouvrage de protection. Elle est menée en deux phases : une analyse du fonctionnement hydro-sédimentaire, indispensable au choix d'hypothèses et de méthodologies adaptées, et une caractérisation des aléas. 


\section{XIV ${ }^{\text {èmes }}$ Journées Nationales Génie Côtier - Génie Civil \\ Toulon, 29 juin au $1^{\text {er }}$ juillet 2016}

\section{2 Échelle et horizon d'étude}

L'échelle d'étude comprend l'ensemble des secteurs susceptibles d'être impactés par le ou les projets, c'est-à-dire l'ensemble de la cellule sédimentaire concernée et l'ensemble des zones inondables affectées par le système de protection étudié, lorsqu'il s'agit d'un projet de gestion des submersions marines. L'impact du changement climatique, notamment l'élévation du niveau moyen de la mer, est non négligeable sur l'horizon d'étude et doit être pris en compte lors de l'étude de mesures de réduction de l'aléa submersion marine. Les ACB, étant classiquement réalisées à aléa constant, doivent dans ce cas prendre en compte la non stationnarité de l'aléa, soit dans le cadre de l'analyse de sensibilité, si l'ACB est réalisé à aléa constant, soit en prenant en compte un aléa non stationnaire (réalisation de plusieurs courbes dommages-fréquences chacune à un niveau marin moyen).

\subsection{Plusieurs niveaux d'analyse en fonction de l'avancement du projet}

L'ACB peut être réalisée à plusieurs stades d'avancement du projet, les analyses des dommages potentiels étant affinés suite à une caractérisation plus fine des aléas.

La première phase de réflexion sur le projet porte sur la stratégie (étude de faisabilité). Parmi l'ensemble des alternatives retenues, elle permet de comparer entre elles plusieurs stratégies d'aménagement ainsi qu'avec la situation de référence. C'est le cas notamment au niveau des stratégies locales de gestion du trait de côte (maintien du trait de côte, relocalisation...) ou des stratégies locales de gestion des inondations.

Une seconde analyse, plus détaillée, s'appuie sur les dimensionnements de l'ouvrage envisagé (études d'avant-projet et projet, le projet déclinant la stratégie) et est utile pour affiner le choix entre deux projets proches et confirmer la pertinence du projet par rapport à la situation de référence. L'analyse est ainsi de plus en plus affinée et précise au fur et à mesure de l'avancement de la démarche de définition des aménagements du fait de l'intégration des résultats d'autres études préalables (étude de dimensionnement, étude de danger).

\subsection{Situations étudiées et coûts associés}

Au démarrage de la démarche de définition de la stratégie d'aménagement, les projets envisagés doivent être comparés grâce à l'ACB à une situation de référence, permettant d'estimer l'intérêt de chaque projet. La situation de référence est celle du maintien de la situation actuelle en continuité des actions présentes. Une stratégie en absence d'ouvrage sur le secteur étudié (pouvant se traduire par un projet de suppression d'un ouvrage existant) est aussi à étudier afin de fournir un second point de comparaison.

Pour chaque situation, il est considéré que le rôle de l'ouvrage est assuré sur l'horizon temporel, les coûts associés sont donc les coûts d'étude et les coûts d'investissement mais aussi les coûts d'entretien, de réparation et de reconstruction éventuelle, établis en prenant en compte la mobilité intrinsèque du trait de côte. 


\section{Analyse du recul du trait de côte}

L'analyse du recul du trait de côte doit être réalisée dans les cas où les aménagements envisagés ont un rôle de limitation du recul ou lorsque le recul peut avoir un impact sur l'aléa de submersion marine, au travers de l'impact sur les cordons dunaires jouant un rôle de protection contre la submersion notamment.

Elle est généralement basée sur l'hypothèse d'un recul du trait de côte linéaire à long terme (en absence de fait marquant une discontinuité dans l'évolution du trait de côte) (voir figure 2).
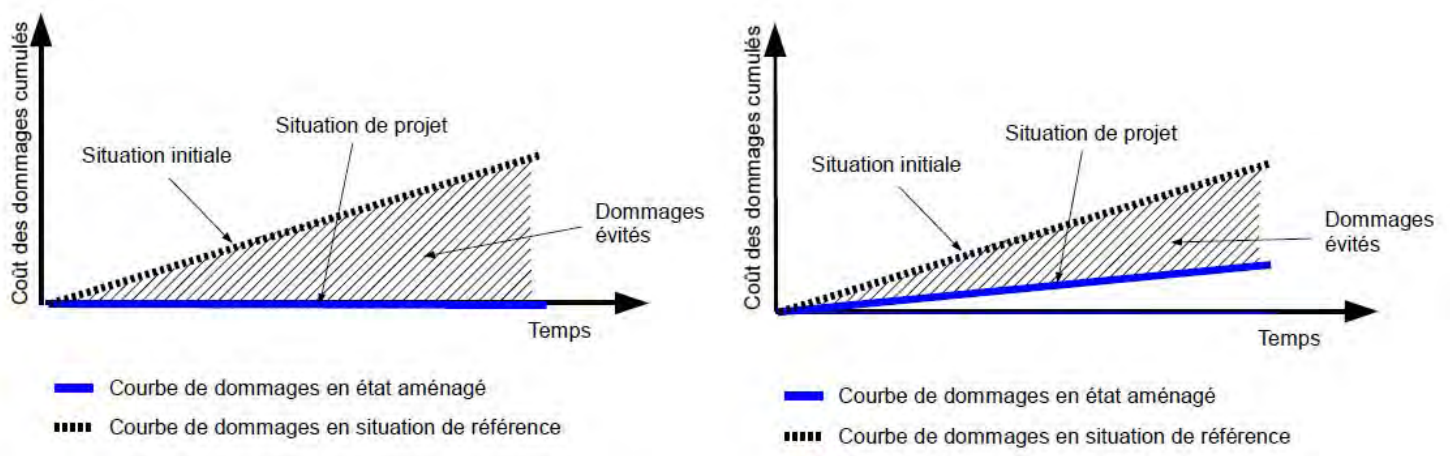

Figure 2. Dommages évités par une mesure (biens conservés), cumulés sur un horizon temporel (à gauche) dans le cas d'un ouvrage fixant le trait de côte et (à droite) ne fixant pas le trait de côte (hypothèse d'un recul du trait de côte et de dommages directement corrélés) (CEREMA, 2015).

\section{Analyse de la submersion marine}

Une courbe dommages-fréquences est réalisée pour chaque situation et pour chaque échéance temporelle (du fait d'un aléa non stationnaire) étudiées. Elle est construite en étudiant plusieurs scénarios d'aléa, correspondant chacun à un événement hydraulique de fréquence donnée et à une hypothèse de prise en compte du comportement de l'ouvrage, permettant chacun de définir un point de la courbe dommages-fréquences. Plus le nombre de scénarios d’aléa étudiés est élevé, mieux on interpole les points pour former la courbe. Les scénarios à étudier de façon privilégiée sont ceux marquant un seuil, ou point anguleux, dans la courbe dommages-fréquences. Les points anguleux de la courbe sont liés aux seuils dans le comportement des ouvrages ou dans la distribution des dommages (fonction de la distribution des enjeux sur le territoire).

$\mathrm{Au}$ stade de définition d'une stratégie de protection, l'ACB permet de comparer différents projets et peut reposer principalement sur l'étude d'une intensité d'événement formant un seuil significatif, correspondant au niveau de protection, lié au comportement de l'ouvrage ou du système de protection. Ce seuil marque des entrées d'eau sur le territoire et est généralement confondu avec le niveau au-delà duquel la tenue de l'ouvrage n'est plus assurée (voir figure 3, gauche). 


\section{XIV èmes Journées Nationales Génie Côtier - Génie Civil \\ Toulon, 29 juin au $1^{\text {er }}$ juillet 2016}

Au stade d'avant-projet, une analyse affinée permet de confirmer l'intérêt d'un projet. Elle se base sur l'étude des scénarios d'aléas correspondant aux points anguleux de la courbe dommages-fréquences, que ceux-ci soient liés au comportement de l'ouvrage (voir figure 3 (droite)) ou à la distribution des dommages. Le comportement de l'ouvrage est principalement défini à partir du niveau de protection, marquant le niveau en deçà duquel l'ouvrage assure l'innocuité des entrées d'eau, et le niveau de sûreté marquant le début de défaillance structurelle de l’ouvrage.
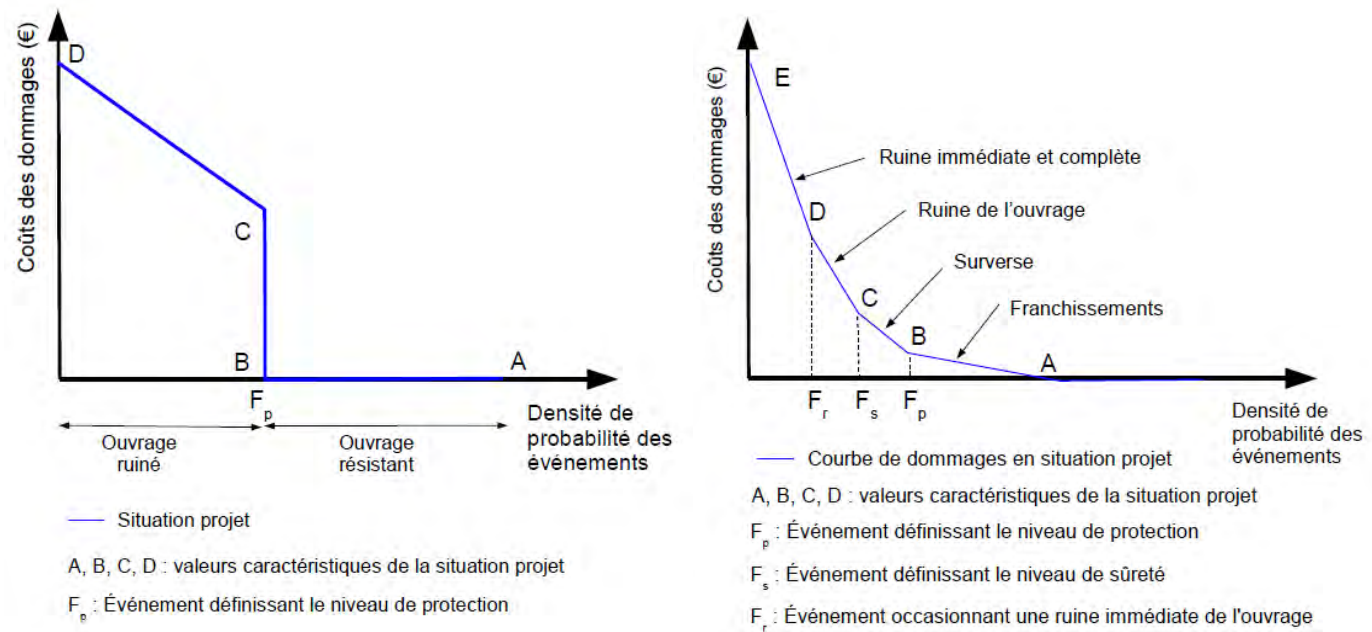

Figure 3. Courbes dommages-fréquences dans le cas d'une analyse simplifiée (à gauche) et affinée (à droite) (CEREMA, 2015).

\section{Estimation des dommages}

Des recommandations spécifiques ont été proposées pour les dommages causés aux logements par la submersion marine du fait du caractère salin de l'eau: emploi des courbes CEPRI de dommages simulant un remplacement systématique des éléments du second œuvre et des équipements fixes.

Dans le cas du recul du trait de côte générant une disparition des biens et activités, une valorisation monétaire de ces biens et activités est proposée (MEDDE, 2014).

\section{Une évolution vers les Analyses Multi-Critères (AMC)}

Si les ACB ont été dans un premier temps le principal outil d'évaluation des mesures de prévention, les analyses multicritères (AMC), dont la méthodologie a été élaborée entre 2012 et 2014 (guide et cahier des charges), pourraient devenir un élément obligatoire du dossier de candidature PAPI car elles permettent une analyse plus complète d'un projet. L'AMC repose sur la confrontation d'indicateurs, qui permettent d'une part de caractériser l'impact du projet sur la réduction des conséquences des inondations sur la santé humaine, l'économie, le patrimoine culturel et l'environnement, et d'autre part de mesurer l'efficacité, le rapport coût-efficacité et l'efficience du projet. Les analyses pré- 
citées contribuent à faciliter la construction de ces indicateurs. La méthodologie AMC a vocation à s'appliquer aux risques littoraux. Des travaux complémentaires sont en cours afin d'adapter cette méthodologie aux spécificités littorales.

\section{Conclusions}

La réalisation d'ACB et d'AMC littorales, réalisées jusqu'alors dans un cadre méthodologique peu adapté, bénéficie aujourd’hui de recommandations spécifiques par le biais du guide Analyse multicritères (MEDDE, 2014) (préconisations pour le traitement des dommages liés aux submersions marines) et du guide Étude des aléas littoraux dans le cadre d'une analyse coûts-bénéfices (ACB) (CEREMA, 2015). Un projet d'annexe portant sur le littoral est envisagé afin d'intégrer les mesures de relocalisation.

Remerciements : Les auteurs remercient les organismes représentés dans le groupe de travail: DGPR, DGALN, CGDD, Cerema, DREAL Poitou-Charente, DREAL Bretagne, DREAL Centre, CEPRI, DREAL Languedoc-Roussillon, ARTELIA, IRSN, EGIS, ISTEA, GIP Littoral Aquitain.

\section{Références bibliographiques}

CEPRI (2011). L'ACB (analyse coût/bénéfice) : une aide à la décision au service de la gestion des inondations. Guide à l'usage des maîtres d'ouvrage et de leurs partenaires, novembre 2011, $44 \mathrm{p}$.

CEREMA (2015). Étude des aléas littoraux dans le cadre d'une analyse coûtsbénéfices (ACB). Collection Références, 52 p.

CGSP -Commissariat général à la stratégie et à la prospective- (2013). L'évaluation socio-économique des investissements publics. Rapports et documents, $352 \mathrm{p}$.

MEDDE (2014). Analyse multicritères des projets de prévention des inondations Guide méthodologique, rapport CGDD, juillet 2014, 86 p.

MEDDTL (2010). Analyse coût bénéfice. Annexes techniques au cahier des charges PAPI, rapport de décembre 2010, 78 p.

MEDDTL, MIOCT, MINEFI, MBCPFPRE. (2011). Plan submersions rapides. Submersions marines, crues soudaines et ruptures de digues, rapport DGPR, 78 p.

MEDDTL (2011). Programmes d'action de prévention des inondations (PAPI) De la stratégie aux programmes d'action. Cahier des charges PAPI, rapport DGPR, 28 p.

MEDDTL (2012). Stratégie nationale de gestion intégrée du trait de côte - Vers la relocalisation des activités et des biens. Rapport MEDDTL/DGALN, 2012, 19 p.

PERHERIN C., CRESPIN N., BOCQUIER L., BARBEAU M.C. (2013). Retour d'expérience de premières analyses coûts-bénéfices littorales. Colloque Digues Maritimes et Fluviales, Aix-en-Provence, pp 624-631. 